\title{
Association between NAFLD and Risk of Colorectal Adenoma in Chinese Han Population
}

\author{
Yuan $\mathrm{Li}^{1}$, Shousheng $\mathrm{Liu}^{3,4}$, Yuqiang Gao ${ }^{1}$, Huan $\mathrm{Ma}^{1}$, Shuhui Zhan ${ }^{1}$, Yan Yang ${ }^{1}$, \\ Yongning Xin*1,2,4 and Shiying Xuan*1,4
}

${ }^{1}$ Department of Gastroenterology, Qingdao Municipal Hospital, Qingdao, China; ${ }^{2}$ Department of Infectious Disease, Qingdao Municipal Hospital, Qingdao, China; ${ }^{3}$ Central Laboratories, Qingdao Municipal Hospital, Qingdao, China; ${ }^{4}$ Digestive Disease Key Laboratory of Qingdao, Qingdao, China

\begin{abstract}
Background and Aims: Colorectal cancer is associated with non-alcoholic fatty liver disease (NAFLD) and other metabolic syndromes, such as obesity, abnormal blood glucose, and dyslipidemia. The relationship of NAFLD and colorectal adenoma, which is the precursor of colorectal cancer, is worthy of discussion. The aim of this study was to investigate the association between colorectal adenoma and NAFLD, colorectal adenoma and metabolic syndrome in a Chinese Han population. Methods: This retrospective study analyzed the relationship between NAFLD and colorectal adenoma in 1089 patients in Qingdao municipal hospital. Subjects were divided into a colorectal adenoma group $(n=267)$ and a control group $(n=822)$. NAFLD and the controlled attenuation parameter (CAP) value were determined by abdominal ultrasound and FibroScan. Results: Patients with NAFLD in the colorectal adenoma group and the control group represented 142 cases (53.2\%) and 360 cases $(43.8 \%)$, respectively. The mean CAP value in the colorectal adenoma group was significantly higher than that in the control group. The values of body mass index, triglyceride, highdensity lipoprotein cholesterol, aspartate aminotransferase, fasting plasma glucose, and uric acid were also significantly higher in the colorectal adenoma group than in the control group. Multifactor logistic regression analysis showed that the sex, NAFLD, CAP, body mass index, triglyceride, aspartate aminotransferase, and fasting plasma glucose were significant risk factors for colorectal adenoma. Besides, NAFLD and CAP value were significant risk factors for colorectal adenoma in males but not in females. Conclusions: NAFLD and metabolic syndrome were tightly associated with the risk of colorectal adenoma in this Chinese Han population. The effect of NAFLD on colorectal adenoma was prominent in males rather than in females.
\end{abstract}

Keywords: Non-alcoholic fatty liver disease (NAFLD); Colorectal adenoma; Metabolic syndrome; Controlled attenuation parameter (CAP).

Abbreviations: ALT, alanine aminotransferase; AST, aspartate aminotransferase; $\mathrm{BMI}$, body mass index; CAP, controlled attenuation parameter; $\mathrm{CRC}$, colorectal cancer; CRA, colorectal adenoma; FPG, fasting plasma glucose; HDL-C, highdensity lipoprotein cholesterol; LDL-C, low-density lipoprotein cholesterol; NAFLD, non-alcoholic fatty liver disease; TC, total cholesterol; TG, triglyceride; UA, uric acid.

Received: 13 February 2019; Revised: 8 April 2019; Accepted: 12 April 2019 *Correspondence to: Shiying Xuan, Department of Gastroenterology, Qingdao Municipal Hospital, 1 Jiaozhou Road, Qingdao 266011, Shandong, China. Tel: +86-532-88905508, Fax: +86-532-88905293, E-mail: xuansydxy@163.com Yongning Xin, Department of Gastroenterology, Qingdao Municipal Hospital, 1 Jiaozhou Road, Qingdao, Shandong 266011, China. Tel: +86-532-82789463, Fax: +86-532-85968434, E-mail: xinyongning@163.com
Citation of this article: Li Y, Liu S, Gao Y, Ma H, Zhan S, Yang $Y$, et al. Association between NAFLD and risk of colorectal adenoma in Chinese Han population. J Clin Transl Hepatol 2019;7(2):99-105. doi: 10.14218/JCTH.2019.00010.

\section{Introduction}

Colorectal cancer (CRC) is one of the most prevailing cancers, with cases having abundant morbidity and mortality every year throughout the world. ${ }^{1}$ According to incomplete statistics, the incidence rates of CRC in males and females rank third and second, respectively, and new cases of CRC are increasing. ${ }^{2,3}$ The 5-year survival rates of CRC patients are reportedly $90 \%$ (stages I-II), $71 \%$ (stage III), and $14 \%$ (stage IV). ${ }^{4}$ As a malignant tumor, CRC progressed from colorectal adenoma (CRA), which is the early stage of CRC. Many factors such as genetic, dietary, and environmental, are known to participate in the occurrence of CRC. 5,6

With economic development, people's living habits and diet structure changes drastically. The metabolic syndromes, such as non-alcoholic fatty liver disease (NAFLD), have become common chronic diseases that endanger human health. Some independent risk factors of NAFLD, such as hyperlipidemia, obesity and diabetes mellitus, have also been identified as potential risk factors for CRC. ${ }^{7-9}$ Accumulated evidence has shown that CRC is tightly associated with NAFLD.

Stadlmayr et al. ${ }^{10}$ investigated the effect of NALFD in patients with colonic neoplasia in Austria by using a crosssectional study, and found that patients with NAFLD showed more CRC precursor lesions and early CRC than the patients without NAFLD. A retrospective cohort study conducted in Korean women by Lee et al. ${ }^{11}$ showed a significant relationship between colorectal neoplasms and NAFLD, and that the NAFLD may act as a predictive risk factor of colorectal neoplasms.

CRA is the precursor of CRC, and the association of CRA with NAFLD had also been studied. Hwang et al. ${ }^{12}$ demonstrated the relationship of CRA and NAFLD by a study conducted in a Korean population for the first time, the results of which indicated that NAFLD was associated with colorectal adenomatous polyps; in addition, an increased risk for NAFLD was observed in the patients with adenomatous polyps. Similar results were observed in the study conducted by Vincent et al., ${ }^{13}$ in which 199 patients with NAFLD and 181 health controls were investigated; the findings indicated 
that patients with NAFLD had a higher prevalence of CRA than the healthy controls, and patients with non-alcoholic steatohepatitis had a higher prevalence of CRA than the patients with simple steatosis. However, Touzin et al. ${ }^{14}$ reported that there was no difference in the occurrence rate of CRA when comparing patients with NAFLD to patients without NAFLD; overall, the results indicated that NAFLD was not an independent risk factor of CRA.

Although some research studies have been carried out to illuminate the relationship of CRA and NAFLD, no related research in Chinese Han population has been reported, and the exact conclusion and detailed mechanism remain to be elucidated.

\section{Objectives}

The aim of this study was to investigate the association of CRA and NAFLD in a Chinese Han population. The NAFLDrelated metabolic indices were also measured to investigate the relationship of CRA with metabolic syndrome.

\section{Methods}

\section{Subjects}

This study was performed in accordance with the Helsinki Declaration and its appendices, ${ }^{15}$ and was approved by the ethical committee of Qingdao Municipal Hospital (Qingdao, China). All the patients enrolled in this study signed an informed consent form before participation in the study.

Subjects were selected from the Department of Gastroenterology of Qingdao Municipal Hospital from June 2015 to March 2018. All subjects were of Northern Han Chinese origin; males and females were included and all were between the ages of 20 and 79 years. All the included subjects had been advised to undergo a colonoscopy to address the following symptoms: abdominal pain, bowel habit change, hematochezia, constipation, iron deficiency anemia, and diarrhea. All the subjects were subjected to colonoscopy carried out by any three experienced endoscopists, each with at least 5 years' experience in colonoscopy examination at the Qingdao Municipal Hospital. Prior to the colonoscopy examination, subjects were given polyethylene glycol electrolyte powder for the bowel preparation. The quality of bowel preparation was graded according to the intestinal residual contents and surface appearance as good (more than $95 \%$ surface seen and without or small volume of clear liquid), poor (less than $90 \%$ surface seen, and semisolid stool presence that could not be suctioned or washed away), and fair (between good and poor). Complete colonoscopy examination was defined as the endoscope (OLYMPUS 260 , Japan) reaching the cecum. Subjects with an incomplete colonoscopy examination were excluded from the study. The CRA was defined as a benign tumor originating from the glandular epithelium of colorectal mucosa, including colon adenoma and rectal adenoma.

Subjects with CRA diagnosed by colonoscopy constituted the CRA group; subjects without CRA were regarded as the control group. All patients were evaluated by medical historytaking and physical examination. Subjects with the following were excluded from the study: 1 ) cancer in the last 2 years; 2) surgery in the last 2 years; 3 ) inflammatory bowel disease; 4) autoimmune disorders (rheumatoid arthritis, autoimmune deficiency syndrome); 5) suffering from several chronic liver diseases (alcoholic liver disease, viral hepatitis, drug-induced liver disease, autoimmune hepatitis) or kidney diseases; 6) having a history of heart failure; 7) suffering from asthma, chronic obstructive pulmonary disorder, or other pulmonary diseases; 8) long-term use of immunosuppressant; or 9) familial colonic polyposis.

\section{Biochemical analyses}

Blood sample were collected from each subject in ethylene diamine tetraacetic acid-containing tubes after a 12-h overnight fast. The body weight and height of each subject were obtained and the body mass index (BMI) was calculated as weight $(\mathrm{kg})$ divided by height $(\mathrm{m})$ squared $\left(\mathrm{kg} / \mathrm{m}^{2}\right)$. For biochemical analyses, blood samples were taken and assessed for serum levels of triglyceride (TG), total cholesterol (TC), high-density lipoprotein cholesterol (HDL-C), low-density lipoprotein cholesterol (LDL-C), fasting plasma glucose (FPG), and uric acid (UA) using routine enzymatic methods in the Roche COBAS8000 Biochemistry Analyzer. Serum aspartate aminotransferase (AST) and alanine aminotransferase (ALT) concentrations were measured by the methods described previously. ${ }^{16}$

\section{Liver ultrasound and controlled attenuation parameter (CAP)}

The liver was scanned by a Siemens Acuson SC 2000 color ultrasound diagnostic instrument and conducted by an experienced sonographer who was blinded to the clinical details of the patients. According to 2010 guidelines for the diagnosis and treatment of NAFLD, patients with viral hepatitis, autoimmune hepatitis, drug-induced hepatitis, various liver cirrhosis and alcoholic liver diseases were excluded. NAFLD diagnostic criteria by ultrasound conformed to (1) the echo intensity of the liver being greater than that of the renal echo; (2) near field echo dense enhancement and far field attenuation; (3) the structure of intrahepatic ducts, especially the veins not being clear; and (4) mild or moderate swelling of the liver. ${ }^{17}$

FibroScan CAP (FibroScan 502 diagnostic instrument, M probe; Echosens Company, France) was measured by three experienced doctors for 10 times at each point, and the CAP median was obtained automatically by the machine.

\section{Statistical analysis}

Statistical analyses were conducted using SPSS 16.0 statistical software. The incidences of NAFLD in patients with CRA and in healthy controls were compared. Meanwhile, the differences of age, sex, CAP, BMI, TG, TC, HDL-C, LDL-C, AST, ALT, FPG, and UA were also compared between the two groups. The biochemical characteristics of the subjects are presented as mean \pm standard deviation or percentages. Categorical variables were compared using the proportions $x^{2}$ test or the Fisher's exact test, as appropriate. Multivariate logistic regression analysis was used to analyze the risk factors of CRA. A $p$-value less than 0.05 was considered statistically significant.

\section{Results}

\section{Characteristics of the study population}

A total of 1410 patients were enrolled before the research, including 370 patients with CRA and 1040 patients without 
CRA. According to the inclusion and exclusion criteria, 103 patients with CRA were excluded because of no liver ultrasound, history of CRC, other extra-intestinal malignancies, viral hepatitis, cirrhosis, history of nephrosis, inflammatory bowel disease, and excessive alcohol consumption. A total of 218 patients without CRA were excluded because of no liver ultrasound, history of operation, viral hepatitis, cirrhosis, pulmonary diseases, arthritis, excessive alcohol consumption, nephrosis, and history of heart disease (Fig. 1). Thus, a total of 1089 subjects were included in the study, consisting of 267 patients ( 175 males and 92 females) with CRA and 822 control subjects ( 391 males and 431 females).

The clinical characteristics of all subjects are shown in Table 1. There were no significant differences for age and the serum levels of TC, LDL-C, and ALT in the CRA group and control group $(p>0.05)$. Compared to the control group, the CRA group had significantly higher BMI $(p<0.001)$, TG $(p<0.001), \operatorname{HDL}-\mathrm{C}(p=0.003), \mathrm{AST}(p=0.003), \operatorname{FPG}(p<$ $0.001)$, and UA $(p<0.001)$.

\section{Prevalence of NAFLD in patients with and without CRA}

NAFLD was diagnosed in 142 of $267(53.2 \%)$ patients in the CRA group and $360(43.8 \%)$ in the control group $(p=0.008)$, and the CAP values in the CRA group were significantly higher than in the control group $(p=0.002)$. Multifactor logistic regression analysis was used to analyze the relationship of CRA with NAFLD, CAP, and lipid metabolism-related factors, and the results showed that sex, NAFLD, CAP, BMI, TG, AST, and FPG were the main risk factors tightly associated with the development of CRA (odds ratio (OR) $=42.170,95 \%$ confidence interval (CI): 21.498-82.720, $p<0.001 ; \mathrm{OR}=$ $1.425,95 \% \mathrm{CI}: 1.112-2.042, p=0.018 ; \mathrm{OR}=1.139,95 \%$
$\mathrm{CI}: 1.047-1.240, p=0.003 ; \mathrm{OR}=1.468,95 \% \mathrm{CI}: 1.199-$ $1.797, p<0.001 ; \mathrm{OR}=1.248,95 \% \mathrm{CI}: 1.003-1.554, p=$ $0.047 ; \mathrm{OR}=1.364,95 \% \mathrm{CI}: 1.275-1.621, p=0.002 ; \mathrm{OR}=$ 1.514, 95\% CI: $1.218-1.882, p<0.001$, respectively) (Table 2).

\section{Characteristics, prevalence of NAFLD in males and females of all subjects}

In consideration of the significant relationship between sex and the risk of CRA, we analyzed the clinical characteristics in males and females of all subjects, respectively (Table 3 ). In males, there were no significant differences in age or the serum levels of TC, LDL-C, and ALT in the CRA group and the control group (all $p>0.05$ ). Compared to the control group, the CRA group had significantly higher BMI $(p<0.001)$, TG $(p<0.001), \mathrm{HDL}-\mathrm{C}(p<0.001)$, AST $(p<0.001)$, FPG $(p<$ $0.001)$, and UA $(p<0.001)$. The occurrence rate of NAFLD and CAP value in males of the CRA group were significantly higher than in males of the control group $(p=0.018 ; p<0.001$, respectively). However, in females, only the serum levels of TG, FPG, and UA were significant different between the CRA group and the control group, and there were no significant differences of the occurrence rate of NAFLD and CAP value between females in the CRA group and females in the control group (all $p>0.05$ ).

Multiple factors logistic regression analysis results showed that NAFLD, CAP values, BMI, AST, and FPG were the independent risk factors of colorectal adenomas in males $(\mathrm{OR}=1.473,95 \% \mathrm{CI}: 1.003-2.162, p=0.048 ; \mathrm{OR}=1.765$, 95\% CI: $1.598-2.068, p=0.002 ;$ OR $=1.833,95 \% \mathrm{CI}$ : $1.409-2.385, p<0.001 ;$ OR $=1.761,95 \%$ CI: $1.452-$ 2.004, $p=0.001 ; \mathrm{OR}=1.511,95 \% \mathrm{CI}: 1.200-1.901$,

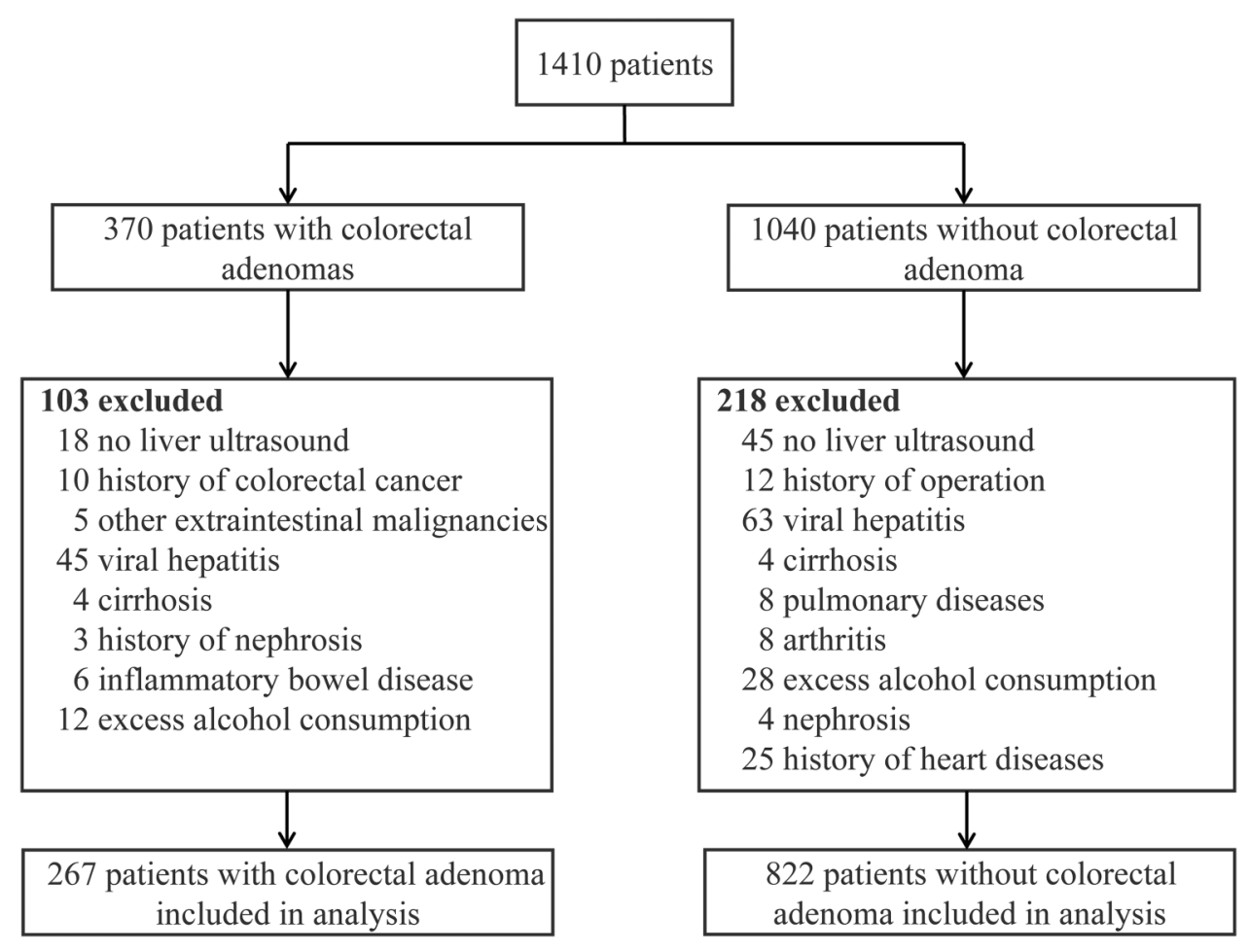

Fig. 1. Flowchart of the study participant recruitment process. 
Table 1. Clinical and biochemical characteristics of patients with or without CRA

\begin{tabular}{llll}
\hline Characteristic & CRA patients, $n=267$ & Controls, $n=822$ & $p$-value \\
\hline Age in years & $54.5 \pm 0.38$ & $54.44 \pm 0.68$ & 0.930 \\
NAFLD & $142(53.2 \%)$ & $360(43.8 \%)$ & 0.008 \\
CAP & $235.56 \pm 30.82$ & $219.12 \pm 29.55$ & 0.002 \\
BMI in $\mathrm{Kg} / \mathrm{m}^{2}$ & $24.54 \pm 1.27$ & $24.22 \pm 0.62$ & $<0.001$ \\
TG in $\mathrm{mmol} / \mathrm{L}$ & $1.99 \pm 1.32$ & $1.45 \pm 0.88$ & $5.46 \pm 1.08$ \\
TC in $\mathrm{mmol} / \mathrm{L}$ & $5.55 \pm 0.98$ & $1.39 \pm 0.32$ & 0.206 \\
$\mathrm{HDL}-\mathrm{C}$ in $\mathrm{mmol} / \mathrm{L}$ & $1.32 \pm 0.37$ & $3.25 \pm 0.82$ & 0.003 \\
LDL-C in $\mathrm{mmol} / \mathrm{L}$ & $3.27 \pm 0.77$ & $29.7 \pm 7.80$ & 0.681 \\
AST in $\mathrm{mmol} / \mathrm{L}$ & $34.5 \pm 8.20$ & $30.3 \pm 9.9$ & 0.003 \\
ALT in $\mathrm{mmol} / \mathrm{L}$ & $31.9 \pm 11.10$ & $4.84 \pm 0.77$ & 0.321 \\
FPG in $\mathrm{mmol} / \mathrm{L}$ & $5.22 \pm 0.99$ & $337.77 \pm 86.01$ & $<0.001$ \\
UA in $\mathrm{mmol} / \mathrm{L}$ & $369.61 \pm 84.26$ & $<0.001$ \\
\hline
\end{tabular}

Abbreviations: NAFLD, non-alcoholic fatty liver disease; CAP, controlled attenuation parameter; BMI, body mass index; TG, triglyceride; TC, total cholesterol; HDL-C, highdensity lipoprotein cholesterol; LDL-C, low-density lipoprotein cholesterol; AST, aspartate aminotransferase; ALT, alanine aminotransferase; FPG, fasting plasma glucose; UA, uric acid.

${ }^{\mathrm{a}}$ Data are presented as mean \pm SD or $n(\%)$.

$p<0.001$, respectively), while the serum levels of TG, HDL-C, and FPG were the independent risk factors of CRA in females $(\mathrm{OR}=1.696,95 \% \mathrm{CI}: 1.254-2.293, p=0.001 ; \mathrm{OR}=2.629$, 95\% CI: $1.106-6.249, p<0.029 ;$ OR $=1.565,95 \% \mathrm{CI}$ : $1.174-2.087, p=0.002$ ) (Table 4).

\section{Discussion}

In this retrospective cohort study, we investigated the relationship of NAFLD and the risk of CRA in both sexes of our study subjects. We observed that NAFLD was more prevalent in the patients with CRA, or in the males among those suffering from CRA (but this was not the case for the females). Similarly, the CAP values and some indices of metabolic syndrome, such as BMI, TG, HDL-C, and FPG, were also significantly changed in the CRA patients, as compared to the controls.

CRC as one of the most prevalent malignant tumors progresses from the tumor precursor of colorectal adenoma. ${ }^{18,19}$ Accumulated evidence has shown that NAFLD is tightly associated with the risks of CRC or CRA, even being regarded as the indicator for colonoscopy among patients of older age. ${ }^{11,20}$ Retrospective and consecutive cohort studies in Chinese, conducted by Lin et al., ${ }^{20}$ showed that NAFLD increases the risk of CRC and that higher severity of malignancy was more common among patients with NAFLD. Huang et al. ${ }^{21}$ reported that in Taipei (China), patients with NAFLD had a higher risk of CRA after negative baseline colonoscopy, and that the NAFLD complications of hyperlipemia and syndrome contributed to the CRA.

The underlying mechanism of the association between NAFLD and CRA is still unclear, but the significant roles of insulin resistance, inflammatory response, and abdominal obesity in the pathophysiological pathways of NAFLD and CRA have been reported. ${ }^{22,23}$ In our study of Northern China (Han population district), we found the occurrence rate of NAFLD to be significantly increased in patients with CRA compared to controls. In addition, the CAP values of all the subjects were measured to investigate hepatic steatosis. Higher CAP values of CRA patients were also observed in comparison to the controls. Multiple factor logistic regression analysis showed that NAFLD and the CAP value were independent risk factors of CRA. These results indicate that the patients with NAFLD or hepatic steatosis had increased risk of CRA.

Sex, as a significant risk factor for CRA, has been reported in some studies. Stadlmayr et al. ${ }^{10}$ reported that male patients with NAFLD had increased risk of tubular adenomas and cancers, while Chen et al. ${ }^{24}$ also reported that NAFLD was the significant risk factor of colorectal adenomatous and hyperplastic polyps in male patients but not in female patients. In our study, we found that sex was a significant factor of CRA. Considering the significance of sex, we analyzed the relationship of

Table 2. Multiple factor logistic regression analysis of the risk of CRA

\begin{tabular}{llll}
\hline Characteristic & OR & $95 \%$ CI & $p$-value \\
\hline Age & 1.004 & $0.988-1.022$ & 0.611 \\
Sex & 42.170 & $21.498-82.720$ & $<0.001$ \\
NAFLD & 1.425 & $1.112-2.042$ & 0.018 \\
CAP & 1.139 & $1.047-1.240$ & 0.003 \\
BMI & 1.468 & $1.199-1.797$ & $<0.001$ \\
TG & 1.248 & $1.003-1.554$ & 0.047 \\
TC & 1.105 & $0.660-1.852$ & 0.704 \\
HDL-C & 0.962 & $0.307-1.329$ & 0.230 \\
LDL-C & 0.906 & $0.504-1.835$ & 0.906 \\
AST & 1.364 & $1.275-1.621$ & 0.002 \\
ALT & 1.123 & $0.786-1.435$ & 0.561 \\
FPG & 1.514 & $1.218-1.882$ & $<0.001$ \\
UA & 1.002 & $1.000-1.004$ & 0.124 \\
\hline
\end{tabular}

Abbreviations: OR, odds ratio; CI, confidence interval; NAFLD, non-alcoholic fatty liver disease; $\mathrm{CAP}$, controlled attenuation parameter; $\mathrm{BMI}$, body mass index; TG, triglyceride; TC, total cholesterol; HDL-C, high-density lipoprotein cholesterol; LDL-C, low-density lipoprotein cholesterol; AST, aspartate aminotransferase; ALT, alanine aminotransferase; FPG, fasting plasma glucose; UA, uric acid. 
Li Y. et al: Association between NAFLD and CRA

Table 3. Clinical and biochemical characteristics of both sexes of patients with or without CRA

\begin{tabular}{|c|c|c|c|c|c|c|}
\hline \multirow[b]{2}{*}{ Characteristic } & \multicolumn{3}{|l|}{ Male, $n=566$} & \multicolumn{3}{|l|}{ Female, $n=523$} \\
\hline & $\begin{array}{l}\text { CRA patients, } \\
n=175\end{array}$ & Controls, $n=391$ & $p$-value & CRA patients, $n=92$ & Controls, $n=431$ & $p$-value \\
\hline Age in years & $53.49 \pm 10.18$ & $53.93 \pm 11.22$ & 0.648 & $56.31 \pm 9.75$ & $55.02 \pm 10.58$ & 0.282 \\
\hline NAFLD & $93(53.1 \%)$ & $166(42.5 \%)$ & 0.018 & $49(53.3 \%)$ & $195(45.2 \%)$ & 0.160 \\
\hline $\mathrm{CAP}$ in $\mathrm{dB} / \mathrm{m}$ & $241.62 \pm 30.57$ & $225.58 \pm 29.12$ & 0.001 & $220.67 \pm 25.56$ & $215.45 \pm 27.68$ & 0.761 \\
\hline BMI in $\mathrm{Kg} / \mathrm{m}^{2}$ & $24.72 \pm 1.14$ & $24.33 \pm 0.63$ & $<0.001$ & $24.20 \pm 1.44$ & $24.12 \pm 0.59$ & 0.371 \\
\hline TG in $\mathrm{mmol} / \mathrm{L}$ & $2.01 \pm 1.25$ & $1.54 \pm 0.96$ & $<0.001$ & $1.94 \pm 1.45$ & $1.38 \pm 0.79$ & $<0.001$ \\
\hline $\mathrm{TC}$ in $\mathrm{mmol} / \mathrm{L}$ & $5.44 \pm 0.96$ & $5.43 \pm 1.06$ & 0.904 & $5.72 \pm 1.09$ & $5.49 \pm 1.10$ & 0.065 \\
\hline $\mathrm{HDL}-\mathrm{C}$ in $\mathrm{mmol} / \mathrm{L}$ & $1.27 \pm 0.30$ & $1.38 \pm 0.33$ & $<0.001$ & $1.43 \pm 0.47$ & $1.41 \pm 0.32$ & 0.694 \\
\hline LDL-C in mmol/L & $3.22 \pm 0.73$ & $3.23 \pm 0.81$ & 0.912 & $3.37 \pm 0.84$ & $3.27 \pm 0.83$ & 0.284 \\
\hline AST in $\mathrm{mmol} / \mathrm{L}$ & $36.70 \pm 11.40$ & $27.60 \pm 10.30$ & $<0.001$ & $33.20 \pm 12.30$ & $31.90 \pm 12.70$ & 0.059 \\
\hline ALT in $\mathrm{mmol} / \mathrm{L}$ & $33.30 \pm 7.80$ & $32.40 \pm 9.20$ & 0.871 & $29.10 \pm 9.30$ & $28.30 \pm 10.90$ & 0.584 \\
\hline FPG in $\mathrm{mmol} / \mathrm{L}$ & $5.18 \pm 0.99$ & $4.85 \pm 0.80$ & $<0.001$ & $5.50 \pm 1.01$ & $4.83 \pm 0.74$ & $<0.001$ \\
\hline $\mathrm{UA}$ in $\mathrm{mmol} / \mathrm{L}$ & $382.97 \pm 84.53$ & $353.38 \pm 84.78$ & $<0.001$ & $343.78 \pm 77.55$ & $323.61 \pm 84.76$ & 0.027 \\
\hline
\end{tabular}

Abbreviations: NAFLD, non-alcoholic fatty liver disease; CAP, controlled attenuation parameter; BMI, body mass index; TG, triglyceride; TC, total cholesterol; HDL-C, highdensity lipoprotein cholesterol; LDL-C, low-density lipoprotein cholesterol; AST, aspartate aminotransferase; ALT, alanine aminotransferase; FPG, fasting plasma glucose; UA, uric acid.

${ }^{\mathrm{a}}$ Data are presented as mean $\pm \mathrm{SD}$ or $n(\%)$.

NAFLD and CAP value with CRA in both male and female subjects. As the results showed, more NAFLD patients and higher CAP values were present in male patients with CRA compared to male controls, but there were no significant differences of NAFLD and CAP value between female patients with CRA and female controls. Multiple factor logistic regression analysis showed that NAFLD and CAP value were the significant risk factors of colorectal adenomas in males but not in the females. Our results were consistent with previous reports of the risk of CRA in males and females being different.
NAFLD is the manifestation of metabolic syndrome in the liver, and is closely related to metabolic factors such as obesity, hyperlipidemia, hyperglycemia, insulin resistance, all of which are also associated with the risk of developing CRC. $8,9,25,26$ BMI is considered the most significant index of obesity, which is mainly caused by excess proteins and fat, such as from red meat. ${ }^{27,28}$ In our study, higher BMI was observed in CRA patients and multiple factor logistic regression analysis showed that BMI was a significant risk factor of CRA. In addition, we observed that BMI was higher in male

Table 4. Multiple factor logistic regression analysis of the risk of CRA in both sexes of subjects

\begin{tabular}{|c|c|c|c|c|c|c|}
\hline \multirow[b]{2}{*}{ Characteristic } & \multicolumn{3}{|l|}{ Male } & \multicolumn{3}{|l|}{ Female } \\
\hline & OR & $95 \% \mathrm{CI}$ & $p$-value & OR & $95 \% \mathrm{CI}$ & $p$-value \\
\hline Age & 1.006 & $0.988-1.024$ & 0.535 & 1.009 & $0.985-1.034$ & 0.449 \\
\hline NAFLD & 1.473 & $1.003-2.162$ & 0.048 & 1.316 & $0.817-2.12$ & 0.259 \\
\hline CAP & 1.765 & $1.598-2.068$ & 0.002 & 1.165 & $0.714-1.461$ & 0.124 \\
\hline BMI & 1.833 & $1.409-2.385$ & $<0.001$ & 1.073 & $0.797-1.443$ & 0.643 \\
\hline TG & 1.200 & $0.952-1.512$ & 0.123 & 1.696 & $1.254-2.293$ & 0.001 \\
\hline TC & 1.127 & $0.632-2.009$ & 0.686 & 0.838 & $0.501-1.402$ & 0.501 \\
\hline HDL-C & 0.532 & $0.234-1.213$ & 0.133 & 2.629 & $1.106-6.249$ & 0.029 \\
\hline LDL-C & 0.917 & $0.445-1.887$ & 0.814 & 1.311 & $0.685-2.508$ & 0.414 \\
\hline AST & 1.761 & $1.452-2.004$ & 0.001 & 1.327 & $0.784-1.754$ & 0.086 \\
\hline ALT & 1.212 & $0.674-1.297$ & 0.154 & 1.132 & $0.934-1.432$ & 0.463 \\
\hline FPG & 1.511 & $1.200-1.901$ & $<0.001$ & 1.565 & $1.174-2.087$ & 0.002 \\
\hline UA & 1.002 & $1.000-1.005$ & 0.065 & 1.001 & $0.998-1.004$ & 0.534 \\
\hline
\end{tabular}

Abbreviations: OR, odds ratio; CI, confidence interval; NAFLD, non-alcoholic fatty liver disease; CAP, controlled attenuation parameter; BMI, body mass index; TG, triglyceride; TC, total cholesterol; HDL-C, high-density lipoprotein cholesterol; LDL-C, low-density lipoprotein cholesterol; AST, aspartate aminotransferase; ALT, alanine aminotransferase; FPG, fasting plasma glucose; UA, uric acid. 
subjects but not in the females; this finding is consistent with the observed effect of NAFLD and CAP value in subjects of both sexes. Higher serum levels of TG, AST, FPG, and UA, and lower serum level of HDL-C were observed in subjects with CRA or in male subjects with CRA. The serum levels of TG, AST, and FPG were identified as significant risk factors of CRA in all subjects. However, the UA level did not significantly vary between the CRA group and the controls, for both female and male subgroups. These results suggest that metabolic syndrome is tightly associated with the risk of CRA in this group and may accordingly act as an indicator for the early diagnosis of CRA.

This study has several limitations. First, the patients with NAFLD were diagnosed by ultrasound rather than by liver biopsy. Second, the underlying mechanism of NAFLD in the risk of CRA was not clear. Third, colonoscopy was performed for diagnostic purposes, different from the routine screening practice for asymptomatic patients and findings of CRA/CRC. Colonoscopy carried out for diagnostic purposes will lead to increased finding of CRA/CRC compared to routine screening. Fourth, this study was conducted in a Chinese Han population, and whether the data are applicable to other ethnicities needs further study.

\section{Conclusions}

In summary, the relationship of NAFLD and the risk of CRA of a Chinese Han population were investigated in this study. We determined that NAFLD was tightly associated with the development of CRA and was the significant risk factor of CRA in all subjects and males but not in females. In addition, we observed that metabolic syndrome had significant effects on the risk of CRA. Although the associations of NAFLD, metabolic syndrome and CRA have been reported previously by many researchers, the detailed mechanisms of how NAFLD and metabolic syndrome affect the development of CRA remains unclear. Further studies with more and different ethnic groups should be conducted to elucidate the underlying mechanism of NAFLD and metabolic syndrome in the development of CRA.

\section{Acknowledgments}

This study was supported by grants from the National Natural Science Foundation of China (No. 31770837) and the Qingdao People's Livelihood Science and Technology Plan (No. 18-6-168-nsh).

\section{Conflict of interest}

The authors have no conflict of interests related to this publication.

\section{Author contributions}

Contributed to study concept and design ( $Y L, Y X, S X)$, acquisition of the data ( $Y L, S L, Y G)$, analysis and interpretation of the data $(Y L, S L, Y G, H M, S Z, Y Y)$, drafting of the manuscript $(Y L, S L)$, critical revision of the manuscript for important intellectual content (YX, SX), and supervision $(Y X, S X)$. All the authors read and approved the final manuscript.

\section{References}

[1] Testa U, Pelosi E, Castelli G. Colorectal cancer: genetic abnormalities, tumor progression, tumor heterogeneity, clonal evolution and tumor-initiating cells. Med Sci (Basel) 2018;6:31. doi: 10.3390/medsci6020031.

[2] Boyle P, Langman JS. ABC of colorectal cancer: Epidemiology. BM] 2000;321: 805-808. doi: 10.1136/bmj.321.7264.805

[3] Sawbridge D, Probert C. Population-based screening in colorectal cancercurrent practice and future developments: faecal biomarkers review. J Gastrointestin Liver Dis 2014;23:195-202. doi: 10.15403/jgld.2014.1121.232.dsw1.

[4] Schreuders EH, Ruco A, Rabeneck L, Schoen RE, Sung J], Young GP, et al. Colorectal cancer screening: a global overview of existing programmes. Gut 2015;64:1637-1649. doi: 10.1136/gutjnl-2014-309086.

[5] Sclafani F, Gullo G, Sheahan K, Crown J. BRAF mutations in melanoma and colorectal cancer: a single oncogenic mutation with different tumour phenotypes and clinical implications. Crit Rev Oncol Hematol 2013;87:55-68. doi: 10.1016/j.critrevonc.2012.11.003.

[6] Budhathoki S, Iwasaki M, Yamaji T, Sasazuki S, Takachi R, Sakamoto H, et al. Dietary heterocyclic amine intake, NAT2 genetic polymorphism, and colorectal adenoma risk: the colorectal adenoma study in Tokyo. Cancer Epidemiol Biomarkers Prev 2015;24:613-620. doi: 10.1158/1055-9965. EPI-14-1051.

[7] Assy N, Kaita K, Mymin D, Levy C, Rosser B, Minuk G. Fatty infiltration of liver in hyperlipidemic patients. Dig Dis Sci 2000;45:1929-1934. doi: 10.1023/a: 1005661516165

[8] Donati G, Stagni B, Piscaglia F, Venturoli N, Morselli-Labate AM, Rasciti L, et al. Increased prevalence of fatty liver in arterial hypertensive patients with normal liver enzymes: role of insulin resistance. Gut 2004;53:10201023. doi: $10.1136 /$ gut.2003.027086.

[9] Jimba S, Nakagami T, Takahashi M, Wakamatsu T, Hirota Y, Iwamoto Y, et al. Prevalence of non-alcoholic fatty liver disease and its association with impaired glucose metabolism in Japanese adults. Diabet Med 2005;22: 1141-1145. doi: 10.1111/j.1464-5491.2005.01582.x.

[10] Stadlmayr A, Aigner E, Steger B, Scharinger L, Lederer D, Mayr A, et al. Nonalcoholic fatty liver disease: an independent risk factor for colorectal neoplasia. J Intern Med 2011;270:41-49. doi: 10.1111/j.1365-2796. 2011.02377.x.

[11] Lee YI, Lim YS, Park HS. Colorectal neoplasms in relation to non-alcoholic fatty liver disease in Korean women: a retrospective cohort study. J Gastroenterol Hepatol 2012;27:91-95. doi: 10.1111/j.1440-1746.2011.06816.x.

[12] Hwang ST, Cho YK, Park JH, Kim HJ, Park DI, Sohn CI, et al. Relationship of nonalcoholic fatty liver disease to colorectal adenomatous polyps. J Gastroenterol Hepatol 2010;25:562-567. doi: 10.1111/j.1440-1746.2009.06117.x.

[13] Wong VW, Wong GL, Tsang SW, Fan T, Chu WC, Woo J, et al. High prevalence of colorectal neoplasm in patients with non-alcoholic steatohepatitis. Gut 2011;60:829-836. doi: 10.1136/gut.2011.237974.

[14] Touzin NT, Bush KN, Williams CD, Harrison SA. Prevalence of colonic adenomas in patients with nonalcoholic fatty liver disease. Therap Adv Gastroenterol 2011;4:169-176. doi: 10.1177/1756283X11402118.

[15] Rickham PP. Human experimentation. Code of ethics of the world medical association. Declaration of Hhelsinki. Br Med J 1964;2:177.

[16] Kotronen A, Westerbacka J, Bergholm R, Pietiläinen KH, Yki-Järvinen H. Liver fat in the metabolic syndrome. J Clin Endocrinol Metab 2007;92:3490-3497. doi: $10.1210 /$ jc. 2007-0482.

[17] Fan JG, Jia JD, Li YM, Wang BY, Lu LG, Shi JP, et al. Guidelines for the diagnosis and management of nonalcoholic fatty liver disease: update 2010 (published in Chinese on Chinese Journal of Hepatology 2010; 18:163 166). J Dig Dis 2011;12:38-44. doi: $10.1111 /$ j.1751-2980.2010.00476.x.

[18] Barbir A, Linseisen J, Hermann S, Kaaks R, Teucher B, Eichholzer M, et al. Effects of phenotypes in heterocyclic aromatic amine (HCA) metabolismrelated genes on the association of HCA intake with the risk of colorecta adenomas. Cancer Causes Control 2012;23:1429-1442. doi: 10 1007/s10552-012-0017-8.

[19] Cross AJ, Ferrucci LM, Risch A, Graubard BI, Ward MH, Park Y, et al. A large prospective study of meat consumption and colorectal cancer risk: an investigation of potential mechanisms underlying this association. Cancer Res 2010;70:2406-2414. doi: 10.1158/0008-5472.CAN-09-3929.

[20] Lin XF, Shi KQ, You J, Liu WY, Luo YW, Wu FL, et al. Increased risk of colorectal malignant neoplasm in patients with nonalcoholic fatty liver disease: a large study. Mol Biol Rep 2014;41:2989-2997. doi: 10.1007/s11033-014-3157-y.

[21] Huang KW, Leu HB, Wang YJ, Luo JC, Lin HC, Lee FY, et al. Patients with nonalcoholic fatty liver disease have higher risk of colorectal adenoma after negative baseline colonoscopy. Colorectal Dis 2013;15:830-835. doi: 10.1111/codi.12172.

[22] Mantovani A, Dauriz M, Byrne CD, Lonardo A, Zoppini G, Bonora E, et al. Association between nonalcoholic fatty liver disease and colorectal tumours in asymptomatic adults undergoing screening colonoscopy: a systematic review and meta-analysis. Metabolism 2018;87:1-12. doi: 10. 1016/j.metabol.2018.06.004. 
[23] Sanna C, Rosso C, Marietti M, Bugianesi E. Non-alcoholic fatty liver disease and extra-hepatic cancers. Int J Mol Sci 2016;17:717. doi: 10. 3390/ijms17050717.

[24] Chen OF, Zhou XD, Sun YJ, Fang DH, Zhao Q, Huang JH, et al. Sex-influenced association of non-alcoholic fatty liver disease with colorectal adenomatous and hyperplastic polyps. World J Gastroenterol 2017;23:5206-5215. doi: 10.3748/wjg.v23.i28.5206.

[25] Benedict $M$, Zhang X. Non-alcoholic fatty liver disease: An expanded review. World J Hepatol 2017;9:715-732. doi: 10.4254/wjh.v9.i16.715.
[26] Machado MV, Cortez-Pinto $\mathrm{H}$. Non-alcoholic fatty liver disease: what the clinician needs to know. World J Gastroenterol 2014;20:12956-12980. doi: 10. 3748/wjg.v20.i36.12956.

[27] Azadbakht L, Esmaillzadeh A. Red meat intake is associated with metabolic syndrome and the plasma C-reactive protein concentration in women. J Nutr 2009;139:335-339. doi: 10.3945/jn.108.096297.

[28] Kim Y, Je Y. Meat consumption and risk of metabolic syndrome: Results from the Korean population and a meta-analysis of observational studies. Nutrients 2018;10:390. doi: 10.3390/nu10040390. 